\title{
Reviewing Product Lifecycle Management Models For Complex Sectors: A Proposal
}

\author{
Angelo Corallo, Mariangela Lazoi, Serena Lettera*, Manuela Marra, Sabrina Verardi \\ Dipartimento di Ingegneria Dell'Innovazione, Università del Salento, Lecce,73100, Italy \\ *Corresponding Author: serena.lettera@unisalento.it
}

Copyright (C) 2014 Horizon Research Publishing All rights reserved.

\begin{abstract}
Recently, the tendency of companies to adopt a PLM (product lifecycle management) strategy is increasing and asks for guidelines and reference frameworks to which screen the own organizational situation and drive improvements. Several scholars and managers have argued about PLM using a business and a technological view. Based on the need of Finmeccanica corporate to provide its companies with a reference guidelines on PLM addressing organizational and technological elements, the paper systematically analyzes models and frameworks available in literature and web-sites. The aim is to provide a set of structured key components useful to guide an exploitation of a corporate associates PLM. Models and frameworks have been organized in three categories (Academic Models, Commercial Models and Enterprise Architecture Frameworks) and fully analyzed. A four-sections PLM model has been specified supported by the evidences coming from literature. The paper provides insights for companies working in complex environment and for theoreticians.
\end{abstract}

Keywords PLM, PLM model, PLM framework, Aerospace and Defence, Aerospace Corporate Company

\section{Introduction}

In companies involved in complex sectors, such as the aerospace \& defense ones, characterized by high technological complexity, high product variety, significant customers participation in product specification, low volume per product and high involvement of stakeholders [1;2], a strategic approach based on product lifecycle management (PLM) provides a systematic view in integrated technologies and methodologies to manage the product data, information and knowledge. Product Lifecycle Management is "an integrated, information-driven approach comprised of people, processes/practices, and technology to all aspects of a product's life, from its design through manufacture, deployment and maintenance - culminating in the product's removal from service and final disposal" [3]. In the last years, the tendency of company to adopt a PLM strategy is increasing and asks for guidelines and reference frameworks to which screen the own organizational situation, highlight gaps and drive improvements. In fact, several authors have argued about PLM using a business or a technological view and they have analyzed and developed models and frameworks creating a large knowledge base distributed on scientific and commercial documents and web-sites.

Generally, a model is a "Graphical, mathematical (symbolic), physical, or verbal representation or simplified version of a concept, phenomenon, relationship, structure, system, or an aspect of the real world. Since most objects and phenomenon are very complicated (have numerous parts) and much too complex (parts have dense interconnections) to be comprehended in their entirety, a model contains only those features that are of primary importance to the model maker's purpose" [4]. It is possible to assume a model as a representation of a real event with its features for satisfying a research scope. Furthermore, based on the research aim, a model on the same "event" can be different to another one.

Therefore, a PLM model wants to provide a synthetic representation reflecting the company practices during a product lifecycle. Different PLM models have been developed representing a focus on specific needs of analysis.

The adoption of a similar PLM models by companies working for the same Corporate society allow to design common initiatives, to underline communalities and differences and to address further adoption of strategies and technologies. The definition of a common model needs, perhaps, the specification of elements and relationships that, led by the sectors and companies peculiarities, integrates best-practices and evidences yet explored and available in the state-of-art.

With these premises, the paper aims to systematically review the PLM models available on scientific literature and on commercial or consulting web-sites for providing a literature review for a Corporate society and its companies. In the specific case, the corporate is Finmeccanica, an Italian holding comprising companies working in the Aerospace \& Defence sector. This research activity has been carried out as an exploratory phase for the activities of the PROLab, a laboratory of the group Finmeccanica in the MindSh@are community of practice, that aims to promote research in 
product lifecycle management and business process management fields.

The paper further wants to propose structured key-elements able to simply represent the landscape of information, data, technologies and their relationships for a product lifecycle. In those companies, PLM plays a strategic role and involves technological and organizational solutions; it supports the management of a big quantity of data and information about product and processes and integrates people and their roles in the whole lifecycle.

PLM features, structure and fields of application have been analyzed from a set of models obtained by a wide internet-based research and organized in three categories based on their source (i.e. academic models, commercial models, enterprise architecture frameworks). The combinations of process, product and technological data and information in the model have been observed as also the structured elements composing the models.

The results of the literature analysis propose a useful reference for companies working in complex environment and suggest elements and relationships for the development of a PLM models common to several companies working for the same corporate. The findings can also be used by theoreticians that could adopt the sets, categories and references reported in the paper to start new researches in the PLM field.

In the next section the literature review and a synthetizes of the analyzed PLM models and framework with some limitations are described. A further section describes the research design. The proposed model is described in a further appropriate section, details on its structure and bibliographical reference are treated. A final section, with conclusions, ends the paper.

\section{Literature Review}

A literature review is based on an interest in a given context and on the need to know what is already known about it [5]. In this study, the literature review has been used to learn about different PLM models, to help the development of its analytical framework and variables to be included. Between the systematic and narrative literature review types, the choice has been in the narrative literature review that allows to have a wider scope to include all the possible relevant contributions for PLM model field [5] and to enrich the human discourse [6] by generating a better understanding on the knowledge of this field.

A recognized weakness of narrative literature review is the lack of systematization of the used criteria but to increase the validity of the realized narrative literature review we have followed the guidelines suggested by Green et al. [7], and the general literature review techniques suggested by Creswell [8]. In the next table 1, a summary of the main steps is provided.

As first step, the key words for the search have been specified and are: "PLM", "Product Lifecycle Model",
"PLM Model", and "Product Lifecycle Management Model". The second step has been to launch several web-based searches to collect all the useful information about models of PLM. In the third and fourth steps of the literature, the different meaning of PLM has been analyzed. In fact, since PLM is also related to the marketing concept of product maturity phases (i.e. introduction, growth, maturity, decline), references with a specific marketing orientation have not been included in the analysis because they are out of the scope of the research that address PLM as a business strategic approach for product lifecycle data and information.

Table 1. Literature Review Steps.

\begin{tabular}{|l|}
\hline (1) Defining keywords for search \\
\hline (2) Searching on electronic databases \\
\hline (3) Highlighting the different meaning for the used keywords \\
\hline (4) Indicating the criteria used to include or exclude an item \\
\hline (5) Providing a literature synthesis with tables or maps \\
\hline (6) Specifying limits \\
\hline
\end{tabular}

A great number of PLM models has been collected. They are available on scientific papers, books, white-papers, consulting or technological-vendor web-sites and private blogs. In the fifth step of the literature review, they have been shared in categories distinguishing between models with a scientific basis and models with a technological or consulting basis. They have been synthetized in forms and table as followed described.

The first category is composed by a wide set of PLM models available in scientific papers of journals or conference proceedings, books or white-papers emerging from theoretical and industrial researches that provide a view on the PLM based on different dimensions. This category has been named Academic Models.

The second category, named Commercial models, involves all the PLM models available on commercial web-sites of IT solutions vendors, consulting firms and thematic blogs; they are simply directed at the promotion of commercial tools or the discussion of different points of view but they provide a PLM vision that is very easy to understand for companies because it is defined for representing the software modules that should be configured in the company.

Furthermore, to provide more information in the PLM understanding a web-based search of the existing Enterprise Architecture Framework (EAF) has been launched. Several frameworks have been evaluated to choice those ones that support the understanding of the company architectural aspects related to the whole product lifecycle and that could be useful to address further insights in the PLM field. This last category, named Enterprise Architecture Framework, is useful to find a source for a same terminology or a set of elements to analyze for a complete view in the organization. 
For each model, a form has been fulfilled. The form is composed by an identification section, a descriptive section and an "innovative notes" section. The first one consists of the name of the model, the authors, the type of source (e.g. conference, journal, book, web-site), and the year of publication or, in the case of web-site, if any other date is not available, the date of access. In the descriptive section, a short summary for each model is realized, describing its scope and dimensions. In the last section, the "innovative notes" one, the distinctive features of the model are reported reiterating the contents of these sections during all the research duration.

The Academic Models category collects the most complete models existing in literature and describes them in their key dimensions. Reading the models description, they have been grouped according to their distinctive elements in six sets: 1) broad scope of PLM, 2) centrality of product data and processes, 3) lifecycle phases, 4) maturity levels, 5) V-model approach and 6) importance of technological layers.

The first set includes the widest models that consider the whole scope of PLM describing all its main components and relations between them. The following two groups (i.e. "centrality of product data and processes" and "lifecycle phases") collect models that emphasize respectively the concept of product data and business processes in PLM implementation and the distinction of several phases in the product development. The fourth set contains models representing the maturity stages in PLM planning and deployment; the fifth group encompasses models that adapt V-model approach to PLM description and finally the models in the last set stress technological solution for PLM.

The Table 2 summarizes the scientific models shared in the specified sets.

PLM models are widely treated in World Wide Web in particular on the web-sites of IT solutions vendors where the PLM views are provided by software vendors for their suite promotion (e.g. Windchill), on web-sites of consulting firms to promote their activities and on thematic blogs with discussions about best practice in PLM. The target of this category of models is a great number of people with different expertise and roles, so they are very simple and easy to understand. The models analyzed are assembled into two different groups: linear PL-phases models and cyclic PL-phases models. The first group includes all the PLM representations where the product lifecycle phases are always shown on a linear axis while the other elements can be different from model to model. An alternative to linear lifecycle phases description is a cyclic representation, which characterizes the cyclic PL-phases models group.

In the Table 3 the analyzed models have been shared in the specified sets.

In this review the last category, Enterprise Architecture (EA) Framework, includes models that support the understanding and awareness of the company architectural aspects related to the whole product lifecycle. They try to put together tools, techniques, artifacts, process models, reference models and all significant issues that revolve around a company. In this review only relevant frameworks for a PLM holistic analysis have been deepened.

The frameworks considered have been collected into two main groups: frameworks focused on the entire organization and its main characteristics and frameworks focused on processes according to an operational point of view. The first group tries to provide a complete exhaustive description of an enterprise through its most important elements; the second group is composed by frameworks useful to depict an enterprise operational level and to analyze different processes.

In the Table 4 the analyzed EAF are summarized.

Table 2. Classification of the PLM models in the academic category.

\begin{tabular}{|c|c|c|}
\hline Category & Set & Reference \\
\hline \multirow{6}{*}{$\begin{array}{l}\text { Academic } \\
\text { Models }\end{array}$} & Broad Scope of PLM & Budde et al. [9]; Silventoinen et al. [10]; Schuh et al. [11]; Rangan et al. [12]. \\
\hline & $\begin{array}{l}\text { Centrality of Product Data } \\
\text { and Processes }\end{array}$ & Abramovici [13]; Sudarsan et al. [14]; Rachuri et al. [15]. \\
\hline & Lifecycle Phases & Terzi et al. [16]; Ameri and Dutta [17]; Hesmer et al. [18]. \\
\hline & Maturity Levels & Buccini et al. [19]; Le Duigou et al. [20]; Batenburg et al. [21]. \\
\hline & V-model approach & $\begin{array}{c}\text { Forsberg and Mooz [22]; US Department of Transportation [23]; Alemanni and } \\
\text { Ciriello [24]; Boehm [25]. }\end{array}$ \\
\hline & $\begin{array}{l}\text { Importance of Technological } \\
\text { Layers }\end{array}$ & Xu et al. [26]; Ming et al. [27]; Chiang and Trappey [28]. \\
\hline
\end{tabular}

Table 3. Classification of the PLM models in the commercial category.

\begin{tabular}{|c|c|c|}
\hline Category & Set & Reference \\
\hline \multirow{2}{*}{$\begin{array}{l}\text { Commercia } \\
1 \text { Models }\end{array}$} & $\begin{array}{l}\text { Linear Product Lifecycle } \\
\text { Phases }\end{array}$ & $\begin{array}{c}\text { AdUltima PLM [29]; Extended Enterprise, [30]; Shilovitsky [31]; Active } \\
\text { Knowledge Modeling [32]; Eigner [33]. }\end{array}$ \\
\hline & $\begin{array}{l}\text { Cyclic Product Lifecycle } \\
\text { Phases }\end{array}$ & $\begin{array}{l}\text { AMR Research [2008] in Phyper and MacLean [34]; Agile PLM Framework } \\
\text { [35]. }\end{array}$ \\
\hline
\end{tabular}


Table 4. Classification of the EAF.

\begin{tabular}{|c|c|c|c|}
\hline Category & Set & Framework Names & Reference \\
\hline \multirow{7}{*}{$\begin{array}{l}\text { Enterprise } \\
\text { Architectur } \\
\text { e (EA) } \\
\text { Framework }\end{array}$} & \multirow{4}{*}{$\begin{array}{l}\text { Frameworks focused on the } \\
\text { entire organization and its } \\
\text { main characteristics }\end{array}$} & Zachman Framework & Zachman International [36] \\
\hline & & $\mathrm{CBM}$ & IBM Consulting Services [37] \\
\hline & & TOGAF v9 & The Open Group [38] \\
\hline & & $\begin{array}{c}\text { Rummler's Process Framework: Value } \\
\text { Creation Hierarchy }\end{array}$ & Rummleret al. [39] \\
\hline & \multirow{3}{*}{$\begin{array}{l}\text { Frameworks focused on } \\
\text { processes according to an } \\
\text { operational point of view }\end{array}$} & $\begin{array}{c}\text { PCF (Process Classification } \\
\text { Framework) APQC and APQC for } \\
\text { Aerospace and Defense }\end{array}$ & APQC [40]; APQC [41]. \\
\hline & & CMMI & CMMI Product Team [42] \\
\hline & & DCOR & Supply Chain Council [43] \\
\hline
\end{tabular}

The analysis conducted and the models studied represent a starting point to provide new insights for the matter of PLM models definition for companies working in complex sectors. The models exploration has been conducted according to the main aim of depicting PLM conceptual elements and their organization in an organic model. However, the study is not completely exhaustive and in the last literature review step some limits and suggestions for future researches have been highlighted. The described analysis has particularly considered models providing a graphical representation of PLM main concepts but to deepen the investigation it could be useful to examine other types of dissertations, i.e. CIMdata [44], which proposed one of the most comprehensive PLM definitions, M. Grieves [3], J. Stark [45], A. Saaksvuori and A. Immonen [46] who wrote books completely dedicated to PLM topic.

In addition, considering the different categories, the research has been developed using key words, and it has resulted in a lot of scientific models but in a few of commercial models. Therefore, for this category further important feedbacks could be provided by major software vendors; it could be useful to analyze different PLM technological implementations in order to understand if there is a PLM vision supporting these solutions and to choose their elements.

Besides, in this review based on the need to represent an holistic PLM, models focused on PLM IT elements have been left out in favor of models representing conceptual elements; therefore, for other scopes of analysis, it could be interesting to examine technological PLM solutions and their links with other tools (e.g. interface between PLM and ERP software).

\section{Research Design}

The study has been carried out with the development of a critical literature review and with the active participation of researchers and key-people involving in the corporate's companies to critical discuss literature and specify elements and structure for a Corporate PLM model. The study is led by the important assumption that PLM implies a strategic approach, and not only an IT system, including different organizational dimensions that need to be evaluated and integrated.

The research has followed an inductive approach based on gaps in the research literature and on the observation of organizational practices, from which the general principles (theories) and solutions have been developed [5].

The literature review has suggested that many of the analyzed models are theoretical or doesn't completely satisfy the needs of PLM model for a group of companies dedicated to the development of complex products. Therefore, the distinctive elements of each of the analyzed models have been combined in order to propose a PLM framework able to cover the needs of complex products lifecycle representations.

With these premises the paper wants to answer at the research question: "Which are the distinctive elements for a complex product lifecycle management? How can they be represented in an integrated framework?"

Using the focus group method [5; 47], each framework have been reviewed between the participants and feedbacks have been collected and organized around a PLM framework proposal commonly shared. In the Figure 1 are summarized the main steps of the research design.

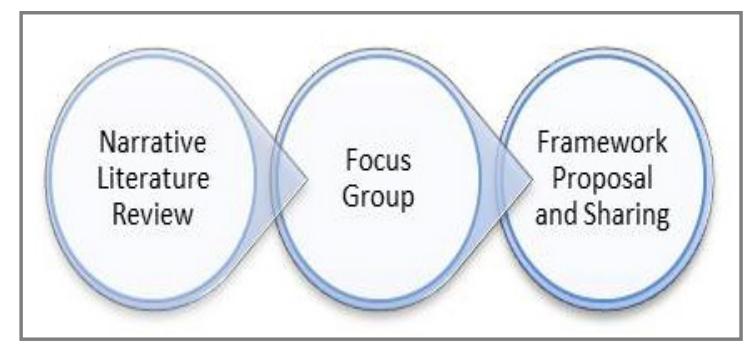

Figure 1. Research Design Phases.

The resulting framework is an useful reference for companies working in complex environment and suggest elements and relationships for the development of a PLM models common to several companies working for the same corporate. 


\section{Results and Discussion}

\subsection{A PLM Conceptual Model}

The characteristic elements of models analyzed in the review are combined in order to propose a new conceptual framework for corporate companies working for the Aerospace and Defence field.

Many companies refer to PLM considering only the IT dimension and ignoring the organizational impact. If it is underestimated, wrong investments and long time for IT deployment can arise. With the proposed Framework, the aim is to provide a pragmatic view around PLM highlighting and representing an organic and integrated structure of processes, activities, data and IT tools related to a specific product lifecycle. The high complexity and diversity of the business of companies working for the same Corporate have led to the design of a solution that can be versatile and adaptable to each involved organization. The Framework therefore is general and of immediate application in different company contexts. Time and complexity depend on the availability of information inside the company.

The proposed PLM Framework is composed by sections and related relational levels: A. PLM Definition and Foundation; B. PLM Phases and Processes; C. PLM Configuration Management Views; D. PLM IT Architecture.

The first element of the framework is a Product Lifecycle
Management definition shared among the Corporate's companies. The PLM definition is impacted by the companies' business and by the realized products/systems. It aims to specify the reference context to which refers the Product Lifecycle Management proposing a product classification that guides the exploitation of the other framework elements.

In the second element of the framework, the main relevance is focalized on two aspects: the lifecycle phases and the processes. The lifecycle phases are the main moments for a product life from its conceptualization to the disposal. Processes are, instead, the sets of activities performed during the phases to obtain outputs relevant for the product, such as components, modules, a specific analysis, a design or a document. The interaction among processes and lifecycle phases is strict. The lifecycle phases evolves thanks to the organizational processes.

A third object of the framework is the set of configuration management views. The configuration management is one of the most relevant processes for companies working in complex sectors, such as Aerospace and Defence. For a company the configuration management views are the perspective of product meta-data during its lifecycle; there are several views of the same product data along the lifecycle phases. Across the working activities in the business processes, product data evolve and are enriched in each lifecycle phase of elements and additional details.

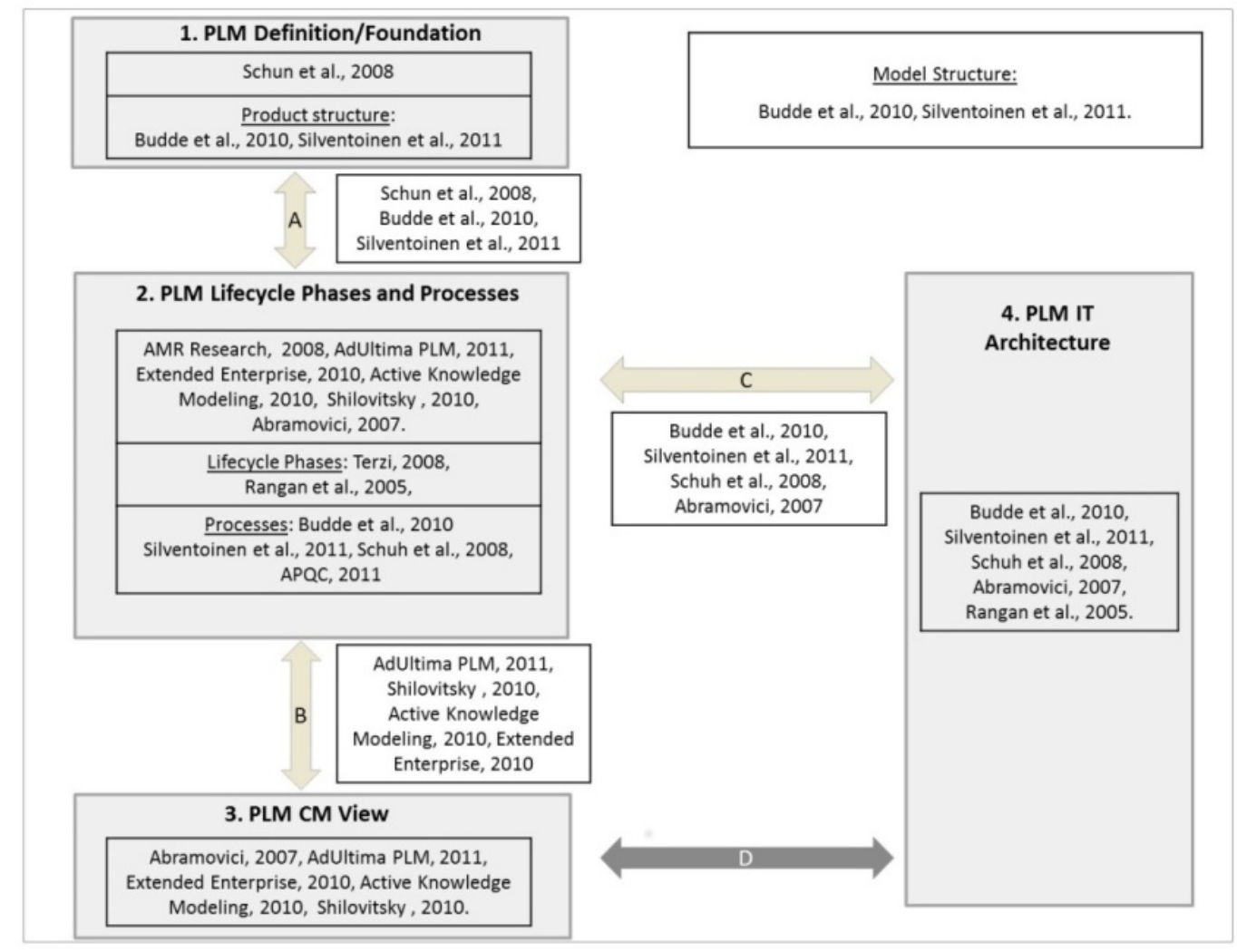

Figure 2. PLM model proposed and its references. 
The fundamental element for product lifecycle management is the technological support. IT tools are the background of all elements being part into PLM. The Framework IT section refers to many heterogeneous IT systems such as document management systems, project management tools, Product Data Management, Customer Relationship Management, Enterprise Resource Management, Collaboration Tool, Workflow \& Change Management, Design Engineering Tools CAx etc. They are used to manage product issues during the lifecycle and for the management of configuration management data. The Figure 2 summarizes the model proposed with the references derived from the review.

With the proposed framework, it is possible to have a PLM reference guide shared among different companies that is actually non-existent. Furthermore, the framework proposes an approach also for technological and processes performance evaluation based on the observation of the real practice and of its implication on the company working.

The framework proposes the relevant elements for the products lifecycle in an integrated structure. Therefore, it could be used to compare different products features and to discover best practices (e.g. in the processes execution) and un-useful replication or application (e.g. in the IT systems use). These lead to adjust inefficient actions and reduce costs.

\subsection{Discussion of the Main References for the Proposed Model}

The main distinctive models investigated in the literature review and their key components for the PLM framework definition are consecutively discussed.

The model structure used for the suggested conceptual PLM model is similar to the model proposed by Budde et al. [9] and Silventoinen [10] analyzed among the papers belonging to the academic model category. These are the most comprehensive models, and define a holistic PLM vision including strategy, culture, processes, product data and IT-architecture aspects. All the other analyzed models focus just on a few of these characteristics. Budde et al. [9] derived a holistic framework for Product Lifecycle Management by analyzing the current literature of integrated management approaches. This model includes four elements important for the development and the implementation of PLM: strategy, process, product structure and IT-architecture. The Strategy dimension focuses on everything related to the creation and safeguarding of the competitiveness of a company (business strategy, product strategy and knowledge management strategy). PLM processes leverage the potential success of a PLM strategy in an operational level; processes are supported by structures. Products structures consist of product platform or families formed as a result of product development processes, and are sustained by organizational structures (e.g. partner network) and information and knowledge structures (e.g. document templates, module library, item naming system or file directory hierarchy). Finally, the technological aspects of PLM are represented by IT-architecture, used in running the PLM processes, in updating of data and information structures. According to the author the challenge is not the selection of single solutions, but the integration of different tools and systems in the holistic implementation.

Budde's model has been discussed by Silventoinen et al. [10], which add a fifth element, people and culture, in order to emphasize the importance of organizational culture and human factor in PLM implementation.

Interrelations between key dimensions are defined both in Budde et al. [9] and Silventoinen et al. [10]. It needs to start with the strategy of an organization: the chosen strategy defines the product development and delivery processes; these processes are intertwined and they need to be well integrated to enable the continuous information flow in the different stages of product lifecycle. The processes are supported by PLM structures and utilize the organizational culture and people as well as the IT architecture as resources.

Budde [2010] and Silventoinen [2011] models, because of their completeness and richness of detail, have been inspired not only the definition of the model structure, but also some specific blocks (PLM Definition and Foundation, PLM Phases and Processes, and PLM IT Architecture) and the relationships among the PLM Phases and Processes block and the PLM IT Architecture one.

The academic model developed by Shuh et al. [11] has guided the definition of the starting point of the PLM framework proposed: the PLM definition block, important in order to establish a clear and unique definition about what is PLM for a firm. They present a process oriented framework comprehending seven elements. The central point consists of a set of lifecycle oriented business process reference models which vary according to a group of company's characteristics (e.g. sector, size, order type, etc.); the set of reference model is linked to other elements: a tangible PLM definition; its foundation, that is the specification of its fundamental concepts; a list of vendor neutral software requirements and specific software solution; a knowledge base; the specification of the potential implementation's benefits.

This model has also inspired the block related to PLM IT Architecture and PLM Phases and Processes, and the connections between this latter block and those named PLM definition and IT architecture.

In addition to Shun many authors provide models based on a process centric vision, for example the Process Driven PLM Framework provided by [34] represents PLM processes as the core features and all the others are developed around them. The structure of this model consists of a central cylindrical element that represents the lifecycle phases in the product development with its supporting organizational elements (i.e. product portfolio, customer needs, collaborative product design, etc.) that is put besides the features of customers and suppliers for PLM. A novelty 
of this model is the collaboration aspect in the product creation process where partners and suppliers work together in the product value creation [34]. This model is proposed in the Cyclic Product Lifecycle Phases model set into the commercial model category and has supported the definition of the second block of the model proposed, PLM lifecycle phases and processes. Between the academic models, lifecycle phases are basic components of Terzi's PLM representation and all the activities related to a product, from its concept phase to its dismission, are grouped into them. Terzi [16] distinguishes three main stages: Beginning of Life (BOL), Middle-of-Life (MOL) and End-of-Life (EOL). The first phase concerns the product design and manufacturing; the second one deals with the real life of the product including distribution, use and maintenance, and the last one is related to product retire and disposal. In the model proposed there isn't this classification, because this could be a decisional parameter for the single firm and for its business context [16].

Also in the Rangan's framework the model structure contains a section dedicated to lifecycle phases. This framework considers 8 key dimensions: Business Drivers, Transformation Drivers, Domain Model, Generic Product Lifecycle, PLM tools, Technology platform, Implementation Drivers and Deployment [12]; it has inspired both the PLM Phases and Processes and the PLM IT Architecture blocks.

In the academic category a notable contribution comes from the Abramovici's model [13]. In this model, the distinctive element is the centrality of business processes and product data. He proposes a representation that includes methods, models and IT tools for managing product information, engineering processes and applications along the different phases of the product lifecycle. He introduces the concept of CM phases linked to the other PLM elements. The insights of Abramovici have been the basis for defining the third block of the model (PLM Configuration Management Views), the block of PLM lifecycle phases and IT Architecture and their relationship.

The main important contributions in the "Commercial Models" category result: AdUltima PLM [29], Shilovitsky [31], Active Knowledge Modeling [32], and Extended Enterprise [30]; they have supported the definition of several blocks of the model, in particular those of PLM processes, $\mathrm{CM}$ views and their connections.

The Windchill Process-led Implementation model [29] offers a simple graphical representation; an axis contains the lifecycle phases and the other one six process typologies (i.e. sales, management, engineering, sourcing, manufacturing, service); twenty-four business processes are mapped according to these two dimensions. The Suite Dassault PLM Model [30] is very similar to the Windchill model; it maps twelve processes grouping them into five different process typologies (i.e. enterprise governance, systems engineering, product engineering, manufacturing, service \& support) and showing their position in lifecycle phases. The Windchill model is more complete than the
Dassault one because it includes the Change \& Configuration Management process, that is one of the most relevant processes in companies working in complex sectors.

The Frank Lillehagen's PLM model described in the Oleg Shilovitsky blog [31] represents the strong integration of different data models. The horizontal axis contains the lifecycle phases, the vertical one defines levels of data and knowledge (i.e. concept, system, component, realization, operations) and different types of data models are linked to these dimensions. The author introduces the concept of User Driven Data Modeling to indicate data models and related views oriented to different roles and scopes (e.g. engineering, manufacturing, etc.).

It is interesting the bi-dimensional graphical view of these three models because they wrap in an easy plan the main elements that participate in PLM [32].

Finally, in the model proposed some characteristics derives from the Enterprise Architecture (EA) Framework analyzed: a primary role is covered by the APQC Framework [41] for its wide specification and classification of processes that can be used to define a common vocabulary in the PLM fields distinguishing among industrial sectors. The APQC Process Classification Framework (PCF), created in 1992, was originally envisioned as a taxonomy of business processes [40]. The PCF organizes operating and management processes into 12 enterprise-level categories, including process groups and over 1,000 processes and associated activities, through its structure and vocabulary. The APQC framework counts from the 1 st to the 3 rd level of detail. APQC has developed industry specific frameworks, so organizations can choose the most suitable for their specific needs. A PCF APQC for the Aerospace and Defense sector is available [41]. Here, the useful interesting element is the opportunity to provide a valuable classification of processes underlying PLM.

The PLM model proposed is characterized by connections among all the elements composing it and, as mentioned above, these features have been stressed by many authors.

The connection between Configuration Management Phases and IT-Systems that manage CM data is missing. The model proposed includes also this link because this connection, applied with the connection Processes-IT tools, could be used to identify information systems used into the product lifecycle (cross D in Figure 1).

\section{Conclusions}

The use of models and frameworks to represent the product lifecycle management is much diffused treating different points of view. In the paper, a wide and complete review of PLM models, emerging from web-based searches, is provided organizing the models in categories and describing the most relevant features and relations.

The review of the PLM models has followed a structured 
approach based on phases that have guided the research and analysis of models. The literature review has followed the guidelines suggested by Green et al. [7] for narrative literature review and the general literature review techniques suggested by Creswell [8]. The models have been shared in three groups (academic models, commercial models and enterprise architecture frameworks) based on the source of the contribution and subgroups based on its main features.

Feedbacks and main contributions of the analyzed models have been combined in the proposal of PLM conceptual model holistic and integrated to be applied by companies of the same Corporate but working in different context. Furthermore, the proposed model is new in the linkage among Configuration Management View and IT system not yet included in other previous works. The sections and linkages are explained and the contributions from the literature review have been highlighted.

For a further pragmatic PLM representation, it could be constructive to follow a bottom-up approach, analyzing different existing examples of PLM implementations belonging to different sectors, for example Aerospace and Defense and Fashion Retail, that present distinct types of complexity.

Therefore, all the analysis done and the emerged considerations are useful to guide future investigations in the PLM fields both oriented to develop industrial specific PLM models and wider research in one or more of the PLM dimensions and directions.

\section{Acknowledgements}

The authors express their appreciation for the executives, managers and development staff of the involved companies.

\section{REFERENCES}

[1] Hicks, C. and McGovern, T. (2009). Product life cycle management in engineer-to-order industries. International Journal of Technology Management, 48 (2): 153 - 167.

[2] Perrone, L. F., Wieland, F. P., Liu, J., Lawson, B. G., Nicol, D. M. and Fujimoto, R. M. (2006). Predicting and improving complex business processes: values and limitations of modeling and simulation technologies. Winter Simulation Conference Proceedings, Monterey, California.

[3] Grieves, M. (2006). Product Lifecycle Management: Driving the Next Generation of Lean Thinking. McGraw-Hill, New York, State.

[4] Business Dictionary. (2011). Available on: http://www.businessdictionary.com/definition/model.html (accessed on 08/05/2012).

[5] Bryman, A. and Bell, E. (2007). Business Research Methods, ed. Oxford University, Oxford.
[6] Geertz, C. (1973). The interpretation of cultures, ed. Basic Books, NY.

[7] Green, B. N., Johnson, C. D. and Adams, A. (2006). Writing narrative literature reviews for peer-reviewed journals: secrets of the trade. Journal Of Chiropractic Medicine, 5: 101 -114 .

[8] Creswell, J. W. (2008). Research Design: Qualitative, Quantitative, and Mixed Methods Approaches, Thousand Oaks, CA, Sage Publications.

[9] Budde, O., Schuh, G. and Uam, J. (2010). Holistic PLM Model - Deduction of a Holistic PLM-Model from the General Dimensions of an Integrated Management. International Conference on Product Lifecycle Management.

[10] Silventoinen, A., Pels, H. J., Kärkkäinen, H. and Lampela, H. (2011). Holistic PLM Model. 8th International Conference on Product Lifecycle Management.

[11] Schuh, G., Rozenfeld, H., Assmus, D. and Zancul, E. (2008). Process oriented framework to support PLM implementation. Comput. Ind., 59(2-3): 210-218.

[12] Rangan, R. M., Rohde, S. M., Peak, R., Chadha, B. and Bliznakov, P. (2005). Streamlining Product Lifecycle Processes: A Survey of Product Lifecycle Management Implementations, Directions, and Challenges. J. Comput. Inf. Sci. Eng., 5 (3): 227-237.

[13] Abramovici, M. (2007). Future trends in product lifecycle management (PLM). The future of product development, ed.F.-L. Krause, Springer, Berlin, 665-674.

[14] Sudarsan, R., Fenves, S. J., Sriram, R. D. and Wang, F. (2005). A product information modeling framework for product lifecycle management. Computer-Aided Design, 37: 1399-1411.

[15] Rachuri, S., Subrahmanian, E., Bouras, A., Fenves, S.J., Foufou, S. and Sriram, R.D. (2008). Information sharing and exchange in the context of product lifecycle management: Role of standards. Computer-Aided Design, 40 (7): 789-800.

[16] Terzi, S., Ball, P. D., Bouras, A., Dutta, D., Garetti, M., Gurumoorthy, B., Han, S. and Kiritsis, D. (2008). A new point of view on Product Lifecycle Management. Proceedings of the International Conference on Product Lifecycle Management, 497 - 528.

[17] Ameri, F. and Dutta, D. (2005). Product Lifecycle Management: Closing the Knowledge Loops. Computer-Aided Design \& Applications, 5: 577-590

[18] Hesmer, A., Eschenbächer, J., Herter, M. and Thoben, K. (2011). Using the Extended Product Concept to better Understand New Business Models along Product Life Cycles: The Case of E-Mobility. International Conference on Product Lifecycle Management. Published on: Interscience Enterprises Ltd.

[19] Buccini, A., Schiraldi, M. M. and Bordi, S. (2011). A conceptual framework to develop assessment models for PLM implementation projects. 8th International Conference on Product Lifecycle Management.

[20] Le Duigou, J., Bernard, A. and Perry, N. (2011). Framework for Product Lifecycle Management integration in Small and Medium Enterprises Networks. Computer-Aided Design \& Applications. 
[21] Batenburg, R., Helms, R. W. and Versendaal, J. (2005). The maturity of product lifecyclemanagement in dutch organizations: Astrategic alignment perspective. Proceedings of the International Conference on Product LifeCycle Management - PLM'05, 436-450.

[22] Forsberg, K. and Mooz, H. (1999). System Engineering for Faster, Cheaper, Better. Proceedings of the ninth annual international symposium of the INCOSE, Brighton, England.

[23] US Department of Trasportation. (2009). System Engineering Guidelines for ITS, Available on:

http://www.fhwa.dot.gov/cadiv/segb/views/process/index.ht m (accessed on 15/11/2011).

[24] Alemanni, M. and Ciriello, D. (2010). PLM in Practice: A methodological approach to drive Implementation. International Conference on Product Lifecycle Management.

[25] Boehm, B. W. (1988). A Spiral Model of Software Development and Enhancement. Computer,21 (5): 61-72.

[26] Xu, X., Fang, S. and Gu, X. (2006). A Framework for Product Lifecycle Management System. International Conference on Management Science and Engineering, 526-530.

[27] Ming, X. G., Yan, J. Q., Lu, W. F. and Ma, D. Z. (2005). Technology Solutions for Collaborative Product Lifecycle Management - Status Review and Future Trend. Concurrent Engineering, 13 (4): 311-319.

[28] Chiang, T. and Trappey, A. J.C. (2006). Development of value chain collaborative model for product lifecycle management and its LCD industry adoption. Int. J. Production Economics, 109: 90-104.

[29] AdUltima PLM. (2011). Available on: http://www.adultima-plm.eu/en/windchill_process_led_impl ementation (accessed on 18/11/2011).

[30] Extended Enterprise. (2010). Available on: http://exenterprise.wordpress.com/ (accessed on 12/11/2011, post 18/12/2010).

[31] Shilovitsky, O. (2010). Available on: Oleg Shilovitsky blog, http://beyondplm.com/2010/05/26/plm-and-user-driven-datamodels/ (accessed on 18/11/2011).

[32] Active Knowledge Modeling. (2010). Available on: http://ac tiveknowledgemodeling.com/2010/05/25/data-modeling-fro m-software-engineering-to-industrial-practice/ (accessed on 18/11/2011).

[33] Eigner, M. (2010). PLM Vision, Future Trends 2010 Conference. Available on: http://global-plm.com/ (accessed on $18 / 11 / 2011$ ).

[34] Phyper, P. and MacLean, P. (2009). (Mis)Management Systems- Chapter 2 in: Good Green Managing Business Risks and Opportunities in the Age of Environmental Awareness, ed.
J. Wiley \& sons, Canada Ltd.

[35] Agile PLM. (2009). Available on: http://www.smashingapps.com/2009/05/06/agility-an-approa ch-to-fight-global-recession.html (accessed on 18/11/2011).

[36] Zachman International. (2006). The Zachman framework, available on: http://www.zachman.com/ (accessed on 16/11/2011).

[37] IBM Consulting Services. (2011). Available on: IBM Consulting Services Website,

http://www935.ibm.com/services/uk/igs/html/cbm-bizmodel. html (accessed on 23/11/2011).

[38] The Open Group. (2009). Available on: http://www.opengroup.org/architecture/togaf9/downloads.ht m\#Non-Member (accessed on 12/11/2011).

[39] Rummler, G. A., Ramias, A. and Rummler, R.A. (2010). White Space Revisited: Creating Value through Process. John Wiley and Sons.

[40] [APQC. (2011). APQC Process Classification Framework Website. Available on: http://www.apqc.org/process-classification-framework (accessed on 28/11/2011).

[41] APQC. (2008). APQC Process Classification Framework for Aerospace and Defense sector. Available on: http://www.apqc.org/knowledge-base/documents/apqc-proce ss-classification-framework-pcf-aerospace-and-defense-pdfversion (accessed on 18/11/2011).

[42] CMMI Product Team. (2010). CMMI for Development, Version 1.3. Available on: http://www.sei.cmu.edu/reports/1 0tr033.pdf (accessed on 23/11/2011).

[43] Supply-Chain Council. (2008). Available on: Supply Chain Council Website, http://supply-chain.org/ (accessed on 28/11/2011).

[44] CIMdata. (2002). Product Lifecycle Management Empowering the Future of Business. Technical report. Available from: http://www.cimdata.com/publications/pdf/PLM_Definition 0210.pdf (accessed 21/12/2011).

[45] Stark, J. (2011). Product Lifecycle Management: 21st Century Paradigm for Product Realisation. Spinger, London.

[46] Saaksvuori, A. and Immonen, A. (2008). Product Lifecycle Management, 2nd ed. Springer, Berlin.

[47] Merton, B.N., Fiske, M. and Kendall, P. L. (1956) The focused interview: a manual of problems and procedures, $2 \mathrm{nd}$ ed., New York, The Free Press. 